\title{
Research Progress in the Functional Textiles
}

\author{
Wei Zhou, Zhaoyi Zhou, and Peng Zhou \\ Shanghai Institute of Quality Inspection and Technical Research, 200040, Shanghai, China
}

\begin{abstract}
This paper reviews the latest research progress of functional textiles at home and abroad. List some of the latest research results and briefly introduce their products.
\end{abstract}

\section{Introduction}

Functional textiles refer to the textiles which not only have their own basic using value, but also have special effects such as anti-bacterial, anti-mite, anti-wrinkle and easy decontamination. Functional textiles can be obtained by applying functional fibers and functional finishing. In recent years, with the improvement of people's consumption level and the diversification of consumption demand, there was a strong demand for functional clothing, home textiles, daily necessities and so on. At present, the research and development of functional textiles would become a fashion and hot spot. International sports brand giants and scientific research institutions are trying to invest huge amounts of money and manpower to seize the commanding heights. The latest research progress of functional textiles in recent years was reviewed.

\section{Safety Protection}

\subsection{Flame retardant protection}

Professor Chen of Donghua University prepared PSA / cellulose flame retardant fiber by dry jet-wet spinning with N-methylmorpholine-N-oxide (NMMO) as cosolvent with polysulfonamide (PSA) and cellulose (cellulose). The unique "skin core" structure of the fiber brought excellent flame retardant performance, and had the moisture absorption, ventilation and comfort of cellulose fiber suitability. ${ }^{[1]}$

Professor Liu of University of science and technology of China synthesized a kind of phosphite modified chitosan (PCS) flame retardant, and deposited the films of PCS and branched polyvinylamine on the polyester cotton blend via layer-by-layer self-assembly technology, so as to prepare polyester fabric with flame retardant property. It was found that the thermal stability of this kind of fabric was enhanced. ${ }^{[2]}$

\subsection{UV Protection}

Professor Wang of Donghua University had prepared a kind of UV shielding agent based on nano- $\mathrm{TiO}_{2}$ and PEG (dispersant) with different relative molecular weight. The anti-ultraviolet finishing of cotton fabric was carried out by dip rolling method. The UPF value of the cotton fabric processed by this process was significantly improved. And the anti-ultraviolet ability of the cotton fabric was effectively improved. ${ }^{[3]}$

Professor Tian of Qingdao University deposited graphene oxide and chitosan on the surface of cotton fiber via layer-by-layer self-assembly technology, and characterized the prepared cotton fiber by SEM, AFM, FTIR and XPS. It was found that the UV resistance coefficient of the modified cotton fiber was 40 times higher than that of the unmodified cotton fiber and then showed excellent UV resistance. ${ }^{[4]}$

\subsection{Biochemical protection}

On the basis of Saratoga chemical protective composite fabric from Germany, Professor Liu of Nantong University selected four different kinds of spherical activated carbon (SAC) as the core adsorption materials, and used the point adhesion technology to composite them with the fabric to form the "anti oil adsorption" mechanism, so as to achieve its protective performance. [5]

Ngoc Bui prepared a thin film based on carbon nanotubes below $5 \mathrm{~nm}$, which could effectively block most virus molecules and poison droplets. At the same time, it could conduct water vapor efficiently. It was an excellent material for semi breathable chemical protective clothing. ${ }^{[6]}$

\subsection{External force impact protection}

Professor $\mathrm{Xu}$ of Manchester University researched on the shear thickening fluid (STF) as a non-Newtonian fluid, when the shear strain rate reached a certain threshold, the viscosity of the fluid would sharply rise to a thousand times to make the fluid transition to quasi solid state, so as to achieve the effect of dispersing the instantaneous impact force and reducing the damage caused by impact. In this study, silica particles and polyethylene glycol 200 (PEG200) were compounded 
with aramid fabric. The anti puncture ability of the modified fabric was significantly improved. And it had higher protection function. ${ }^{[7]}$

British PPSS group had developed a kind of sanitary clothes based on the patent Cut-Tex®Pro, which combined UHMWPE and special ultra-high molecular materials. Its wear resistance, cutting resistance and tear resistance met the highest level of European standards. The new Cut-Tex®EXTREM product of the company could provide a higher level of protection while its thickness was almost the same as that of ordinary $\mathrm{T}$ shirts.

\section{Comfort Function}

\subsection{Antistatic}

Professor Hassan of Yamagata University introduced silver nanoparticles and trisodium citrate into acrylic fibers. By adjusting the ratio of silver nanoparticles and trisodium citrate, acrylic fibers showed excellent versatility. The introduction of silver nanoparticles could reduce the resistance of acrylic fiber and had excellent antistatic performance, which was conducive to the application of new acrylic fiber. ${ }^{[8]}$

Professor Zhang of Tianjin University of Science\&Technology prepared a kind of antistatic polyaniline modified wool fiber by in-situ adsorption polymerization. It was found that polyaniline was formed by in-situ self-assembly on wool fiber, uniformly dispersed and tightly coated on the surface of wool fiber. At the same time, the resistance value of the modified wool decreased and remained unchanged after several times of washing, which indicated that the modified fiber had good and stable antistatic property. ${ }^{[9]}$

\subsection{Waterproof and moisture permeable}

Professor Hu of Hong Kong Polytechnic University had obtained a kind of smart composite film by blending silica aerogel particles (SAs) into thermo humidity sensitive thermoplastic polyurethane (THSPU), showing excellent waterproof performance. At the same time, under the change of temperature and humidity, the moisture permeability would change accordingly and showed a typical adaptive moisture permeability function. The introduction of SA structure could also bring excellent thermal insulation properties of the composite film. The above-mentioned adjustability based on environmental change was conducive to the management of human "microclimate", which was expected to promote the development of smart clothing and wearable products, shown in Fig. $1^{[10]}$

Professor Luo of Shanghai University reported a method for the preparation of pure polyvinylidene fluoride (PVDF) superhydrophobic microporous membrane based on high humidity induced electrospinning process. The superhydrophobic property of PVDF microporous membrane was determined by its special microsphere fiber interpenetration structure. By adjusting the concentration of PVDF in polymer solution, the effective pore size and porosity of PVDF membrane could be well adjusted, so as to improve the waterproof and air permeability of the membrane. ${ }^{[11]}$

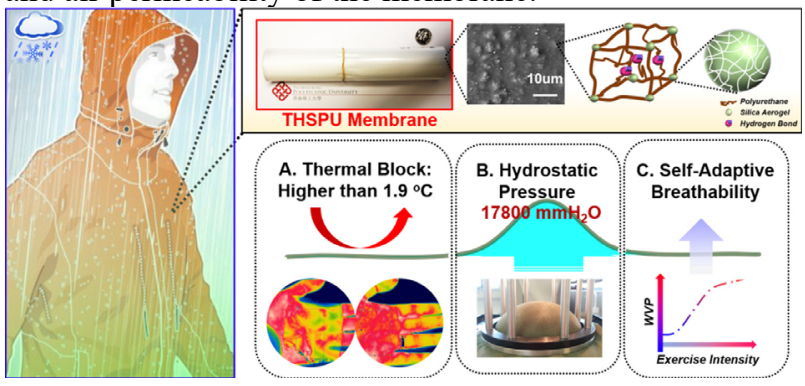

Fig. 1. Function and mechanism of self-adaptive breathable polyurethane membrane ${ }^{[10]}$

\section{Intelligent Textiles}

\subsection{Intelligent temperature regulation}

Professor Zhang of the University of Maryland, based on a special fiber with trace carbon nanotubes, had excellent infrared radiation "gating" effect under changing environmental conditions, shown in Fig $2^{[12]}$. Under different temperature and humidity conditions, the arrangement of sand lines in the fabric was closely arranged, which leads to the change of the distance between conductive materials and leads to the electromagnetic coupling between conductive materials, which makes the infrared radiation transmittance increase. Finally, it showed the intelligent control of body temperature.

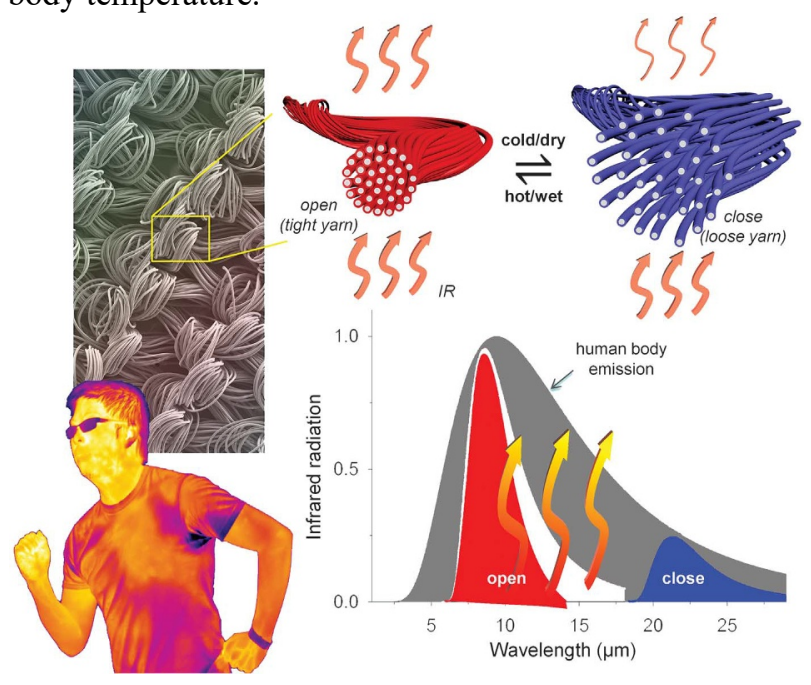

Fig. 2. "gating" effect of infrared radiation intelligent fabric ${ }^{[12]}$

On the basis of skin friendly silk protein, Professor $\mathrm{Wu}$ of Huazhong University of Science and Technology designed and controlled the internal structure of the fiber by freezing spinning method, and prepared a kind of light, waterproof, soft and comfortable intelligent temperature regulating fabric. Its intelligent temperature regulation performance mainly came from the integrated temperature control phase change material coated with PDMS coating in the fiber structure, which had the effect of absorbing, storing and releasing energy. In the test 
process, it was found that this kind of fabric could absorb energy from the environment at high temperature to cool the skin covered by the fabric. At the same time, when the surrounding environment of the fabric was reduced, it could release heat correspondingly, indicating the formation of its intelligent temperature regulation property for human body temperature. ${ }^{[13]}$

\subsection{Shape memory}

Professor Narayana of Hong Kong Polytechnic University designed and manufactured a kind of medical compression socks based on shape memory polyurethane fiber, shown in Fig. $3^{[14]}$. It was found that the higher the stress and the higher the temperature, the stronger the stress memory property of the material. This kind of compression socks with shape memory could help venous blood circulation, which had a wide application potential in the medical field.

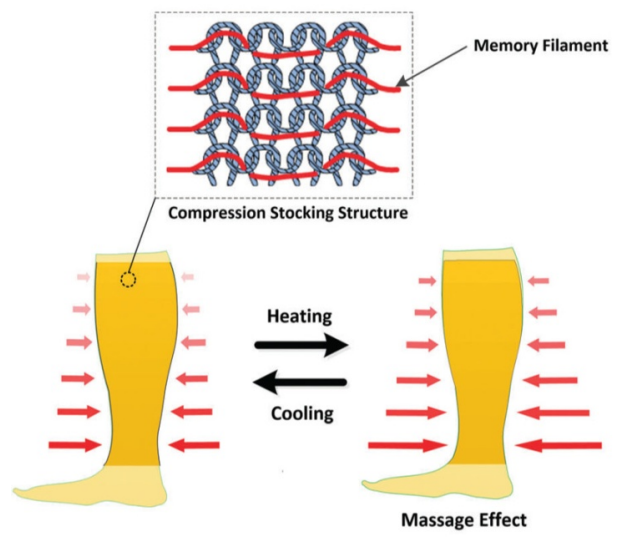

Fig. 3. Schematic of shape memory socks ${ }^{[14]}$

Professor Bai of Donghua University had successfully grafted N-isopropylacrylamide (NIPAAm) onto reduced wool macromolecules based on thiol olefin click chemistry. Through the characterization, it was found that this kind of method that the group with shape memory effect was inserted into the original fabric structure by click chemical reaction could effectively endow the wool fabric with shape memory characteristics. At the same time, it could effectively improve the hydrophilicity of wool fabric. This study provided a new perspective for the preparation of smart textiles from the perspective of synthesis methods. ${ }^{[15]}$

\subsection{Intelligent color change}

A kind of urea formaldehyde microcapsule with photosensitive yellowing dye as core material was prepared by in-situ polymerization by Professor Xue of Jiangnan University, and it was attached to cotton fabric by coating process. By adjusting the mass fraction of microcapsules, the best washing and color changing properties of the fabric were obtained. The final fabric could reach the maximum color change after 30 seconds of sunlight, and the fabric had good color change effect after this finishing technology. ${ }^{[16]}$

Professor Li of Donghua University dip coated chemically reduced graphene ( $\mathrm{RGO}$ ) - $\mathrm{TiO}_{2}$ conductive layer, PDMS protective layer and thermochromic ink layer on the basis of double-layer stretch elastic core spun yarn (dcys). The fiber could cause temperature rise in the process of drawing, which made the thermochromic ink change color, which was the fabric color change. By changing the type of thermochromic ink, the color change of fabric could be realized. Through optimization, the research group had achieved the continuous preparation of ultra long electrochromic fibers, shown in Fig. $4^{[17]}$, laying a solid foundation for the industrial preparation of such intelligent color changing fabrics.

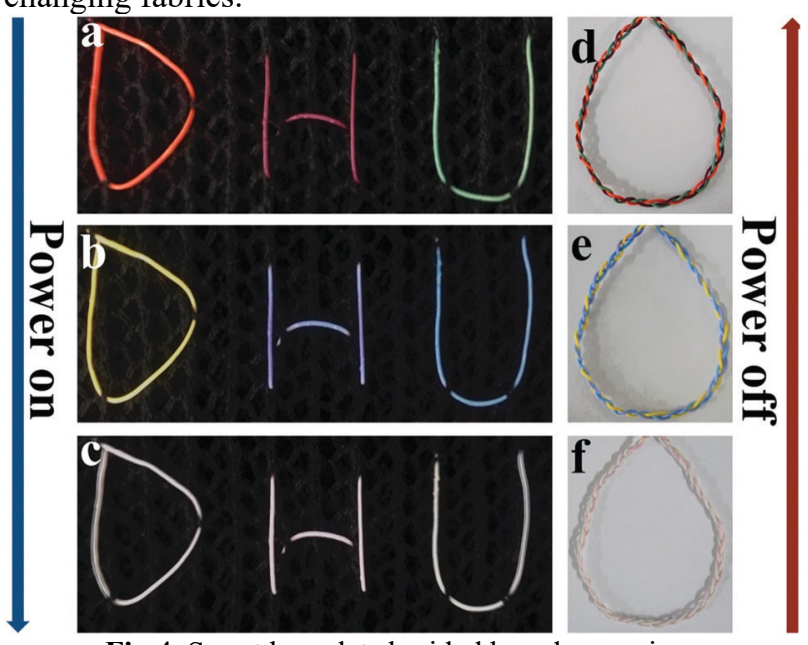

Fig.4. Smart bracelets braided based on various electrochromatic fibers ${ }^{[17]}$

\subsection{Electronic fabric}

Professor Liu of Beijing Institute of nano energy and systems, Chinese Academy of Sciences, achieved the effect of large area pressure sensor on the preparation of ordinary fabrics by fusing the conductive pattern of $\mathrm{Ni}$ coated polyester fiber with carbon nanotube cotton fabric. Through the detection of the stress distribution of various parts of the body by these electronic components, the real-time monitoring and response of physiological activity health were achieved, shown in Fig. 5. ${ }^{[18]}$

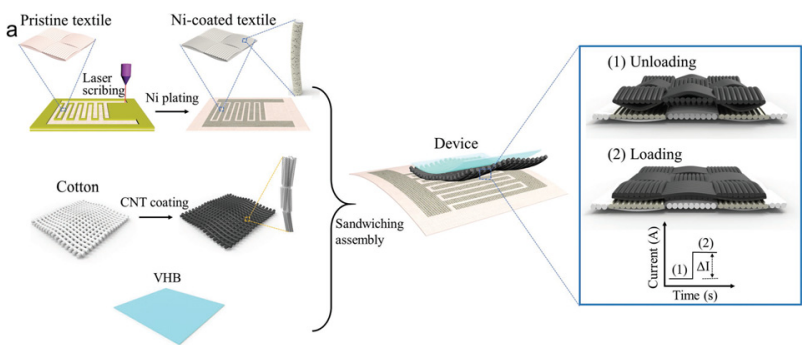

Fig. 5. Schematic of the fabrication procedure of textile pressure sensors. ${ }^{[18]}$

Professor Chai of Jinan University had woven the fiber-shaped dye-sensitized solar cells and fiber-shaped supercapacitors to form an intelligent wearable fabric. When the fabric was exposed to light, solar cells and capacitor modules work together to convert solar energy into electricity and store it. It was worth noting that this kind of fabric had tailorability because of its structure. It 
could be cut into any shape and keep its energy collection and energy storage properties. It provided a new idea for wearable intelligent clothing. ${ }^{[19]}$

\section{Health Care}

\subsection{Antibacterial type}

Through free radical polymerization, Professor Han of Donghua University synthesized nano hydrogels with durable antibacterial and bacterial adhesion by one-step method. The material had antibacterial guanidine segments and hydrophobic polycaprolactone chains, which could effectively prevent bacteria from adhering to the surface of cotton fabrics. The nanogels quickly killed the bacteria that contact them by destroying cell membranes and causing cell lysis. Compared with the traditional antibacterial nano hydrogel materials, the antibacterial ingredients in monomeric form had long lasting antibacterial effect, good biocompatibility, strong bactericidal ability and had a positive effect on preventing wound infection. At the same time, the nano hydrogel could be grafted on the surface of cotton fabric, so that the cotton fabric itself had the function of durable antibacterial and bacterial adhesion, and could effectively prevent bacteria from propagating and proliferating on cotton fabrics. The thermodynamic stability of the nano hydrogel enabled it to be applied to various environments in the process of fabric finishing, and opened up a new direction for antibacterial finishing of clothing. ${ }^{[20]}$

There were two kinds of natural antibacterial mode: Natural antibacterial fiber and natural antibacterial agents. Natural antibacterial fiber had good antibacterial properties because of its own fiber structure or natural antibacterial substances which could inhibit and kill bacteria. Natural antibacterial fibers mainly included chitosan fiber, chitin fiber, hemp fiber, bamboo fiber, tea fiber, kapok fiber, etc. Natural antibacterial agent was mainly from the active substances extracted from plants and animals to finish the fabric, so as to achieve the antibacterial properties of the fabric. There were mainly two kinds of antibacterial agents, which were mainly composed of chitosan and chitin, and flavonoids, anthraquinones, polyphenols, alkaloids and positively charged plant antibacterial agents. ${ }^{[21]}$

\subsection{Anti-mite}

Professor Huang of Beijing Tongniu Group Co., Ltd. had developed bamboo cotton sweatcloth in spring/summer and graphene inner warm elastic double rib fabric in autumn/winter by using natural antibacterial and anti mite function of Tianzhu fiber. After testing, it was found that the two fabrics had excellent antibacterial and anti mite effects. ${ }^{[22]}$

Professor Wu of Guangzhou Infinitus company had the anti-mite properties of microcapsules made from traditional Chinese medicine Litsea cubeba and cumin and the microcapsules made from thyme essential oil. A modified viscose fiber was obtained from wet spinning of viscose fiber blend. Through the characterization test, it was found that the anti-mite performance of this kind of viscose fiber was significantly improved before and after modification. ${ }^{[23]}$

\subsection{Far-infrared fiber}

Professor Gao of Suzhou University produced a kind of far-infrared polyester fiber by masterbatch method using two kinds of far-infrared materials (nano- $\mathrm{ZrC}$ and $\mathrm{Al}_{2} \mathrm{O}_{3}$ ). It was found that the temperature and heating rate of the fabric were obviously improved under infrared irradiation. At the same time, the contact cold feeling and heat conductivity of the fabric were low, which indicated that the heat preservation performance of the fabric was good. ${ }^{[24]}$

Professor Gong of Guangdong Dymatic Chemical Group Co., Ltd. used a kind of far-infrared functional powder elite-a with thermoelectric effect and piezoelectric effect to finish various kinds of existing textiles by coating method and dip rolling method. By changing the ratio of raw materials, the optimal ratio of far-infrared functional powder, thickener and adhesive for the far-infrared heating efficiency and washing resistance of the fabric was obtained, which provided the basis for the actual production of far-infrared functional textiles. ${ }^{[25]}$

\section{Summary}

In conclusion, functional textiles have become an emerging field with high technology content, high industrial added value and large market volume. With the continuous improvement of people's requirements for safety, comfort and experience, the application field of functional textiles is also expanding now, which is particularly urgent to meet the needs of market development.

\section{References}

1. T. Cheng, Y. Yao, Z. Chen, et al. Journal of Textile Research, 040(007), 1-7, (2019)

2. L. Liu, Y. Pan, Z. Wang, et al. IND ENG CHEM RES, acs.iecr.7b02303, (2017)

3. L. Wang, Y. Zhang, Z. Cai, Technical Textiles, 36(10), 43-45, (2018)

4. M. Tian, X. Hu, L. Qu, et al. APPL SURF SCI, 377, 141-1481, (2016)

5. Q. Liu, Y. Zhou, Z. Yang, et al. Journal of Textile Research, 40(06), 183-189, (2019)

6. N. Bui, E.R. Meshot, S. Kim, et al. Adv Mater, 28(28), 5871-5877, (2016)

7. Y. Xu, X. Chen, Y. Wang, \&Z. Yuan, Compos Struct, 163, 465-473, (2017)

8. M.M. Hassan, K. Kiyohito, Dyes Pigments, 159, 517-526, (2018) 
9. H. Zhang, J. Yang, Z. Wu, et al. J Tianjing Uni Sci Technol, 4, 42-44, (2019)

10. S. Shi, Y. Han, J. Hu, Prog Org Coat, 137, 105303, (2019)

11. G. Jiang, L. Luo, L. Tian, et al. ACS Appl Mater Inter, 10, 28210-28218, (2018)

12. X. Zhang, S. Yu, B. Xu, et al. Science, 363(6427), 619-623, (2019)

13. J. Wu, R. Hu, S. Zeng, et al. ACS Appl Mater Inter, 12, 19015-19022, (2020)

14. H. Narayana, J. Hu, J. Han, S. Shang, et al. J Mater Chem B, 5, 1905, (2017)

15. S. Bai, W. Wang, D. Yu. Wool Textile Journal, 47(03), 22-25, (2019)

16. Q. Xue, T. Wang, F. Sun, et al. Textile Auxiliaries, 5, 18-22, (2018)

17. Q. Li, K. Li, H. Fan, et al. J Mater Chem C, 5, 11448, (2017)

18. M. Liu, X. Pu, C. Jiang, et al. Adv Mater, 29(41), 1703700.1-1703700.9, (2017)

19. Z. Chai, N. Zhang, P. Sun, et al. ACS Nano, 10, 9201-9207, (2016)

20. H. Hua, J. Zhu, D. Wu, et al. Adv Funct Mater, 29(12), 1806594, (2019)

21. H. Zhang, X. Zhang, M. Liu, et al. Progress in Textile Science \& Technology, 3, 8-11, (2020)

22. X. Huang, L. Chen, Y. Wu, et al. Knitting Industries, 6, 27-30, (2018)

23. J. Wu, H. Yu, X. Wan, et al. Journal of Textile Research, 40(007), 19-23, (2019)

24. B. Gao, Y. Xu, X. Guan, et al. Shanghai Textile Science \& Technology, 392(02), 24-28, (2016)

25. J. Gong, X. Gu, J. Xiao, et al. Knitting Industries, 358(11), 84-86, (2018) 